\title{
3,4,5-Trihydroxycinnamic Acid Inhibits LPS-Induced Inflammatory Response by Increasing SIRT1 Expression in Human Umbilical Vein Endothelial Cells
}

\author{
Jin-Young Park ${ }^{a}$ Hee Jae Lee ${ }^{a}$ Yong-Soo Kwon $^{b}$ Wanjoo Chun ${ }^{a}$

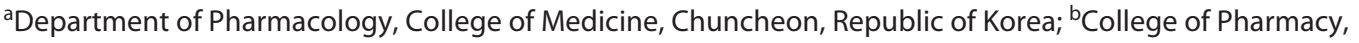 \\ Kangwon National University, Chuncheon, Republic of Korea
}

\section{Keywords}

3,4,5-Trihydroxycinnamic acid - Sirtuin 1 - p53 - Intercellular adhesion molecule-1 - Vascular cell adhesion molecule-1 . Lipopolysaccharide $\cdot$ Human umbilical vein endothelial cells

\begin{abstract}
3,4,5-Trihydroxycinnamic acid (THC) has been demonstrated to exert anti-inflammatory activities in LPS-induced RAW264.7 murine macrophage cells and in LPS-induced septic mice. However, the effect of THC on the inflammatory response in vascular endothelial cells has not been clearly examined. The goal of the present study was to elucidate the anti-inflammatory properties of THC and its underlying mechanism in LPS-challenged human umbilical vein endothelial cells (HUVECs). THC significantly suppressed LPS-induced interleukin-1 $\beta$ production and intercellular adhesion molecule-1 and vascular cell adhesion molecule- 1 expression and significantly decreased LPS-induced nuclear factor$\mathrm{KB}$ activation by attenuating $\mathrm{p} 65$ phosphorylation and inhibitor of kappa $B$ degradation. To understand the underlying mechanism of the anti-inflammatory effect of THC, the involvement of the sirtuin 1 (SIRT1) signaling pathway was ex-
\end{abstract}

amined. THC resulted in increased expression of SIRT1 in LPS-challenged HUVECs. Among the downstream molecular targets of SIRT1, the level of LPS-induced acetylated p53 was significantly decreased by THC treatment, whereas no noticeable change was observed in the levels of forkhead box $\mathrm{O} 3$ and peroxisome proliferator activated receptor gamma coactivator 1 alpha. In conclusion, the results clearly demonstrate that THC possesses anti-inflammatory properties by increasing SIRT1 expression and subsequent suppression of p53 activation in LPS-challenged HUVECs.

(c) 2020 S. Karger AG, Basel

\section{Introduction}

Endothelial cells produce a wide range of inflammatory mediators that exacerbate endothelial dysfunction in inflammatory conditions [1]. Endothelial malfunction caused by inflammation plays a dominant role in the pathogenesis of various vascular disorders, including hypertension, atherosclerosis, and diabetes-induced vascular disorders $[2,3]$. Previous reports strongly suggest that endothelial cells might play a key role in the pathophysiology of sepsis - a severe systemic inflammatory reaction

$\begin{aligned} & \text { karger@karger.com } \\ & \text { www.karger.com/jvr }\end{aligned}$
Karger ${ }^{\prime /}$

Dr. Wanjoo Chun

Department of Pharmacology, College of Medicine Kangwon National University

Hyoja-2, Chuncheon 24341 (Republic of Korea)

wchun@ kangwon.ac.kr 
to infection that is caused by aberrant stimulation of the host immune response by pathogenic components [4]. The pathogenic role of endothelial activation and dysfunction in sepsis was clearly demonstrated in the abnormal upregulation of cell adhesion molecules, which are proteins involved in endothelial-related adhesion and coagulation $[5,6]$. This resulted in an increase in leukocyte trafficking, and in coagulation, vascular permeability, and inflammation, which are the key characteristic features of sepsis [7]. It has been reported that cell adhesion molecules and pro-inflammatory mediators are upregulated through the activation of the nuclear factor kappalight-chain-enhancer of activated B cells (NF- $\mathrm{B}$ ) signaling pathway $[8,9]$. Therefore, attenuation of aberrant vascular endothelial activation and pro-inflammatory mediator expression may have significant therapeutic potential for the treatment of inflammation-induced endothelial dysfunction.

Sirtuin 1 (SIRT1), a member of the sirtuin protein family, is an NAD-dependent deacetylase protein [10] and is involved in the regulatory control of diverse cellular processes, such as cell survival, apoptosis, DNA repair, autophagy, and cell migration, via deacetylation of various intracellular proteins $[11,12]$. SIRT1 is known to regulate the activity of downstream molecular targets such as cellular tumor antigen p53, forkhead box O3 (FOXO3a) protein, and peroxisome proliferator-activated receptor gamma coactivator 1 alpha (PGC1 $\alpha$ ) via deacetylation modification [13]. Acetylation plays an important role in the activation of $\mathrm{p} 53$, which is a major stress regulator that responds to apoptotic cell death. Acetylated p53 induces apoptosis through the mitochondria-dependent pathway $[14,15]$. It has been reported that enhanced expression of SIRT1 significantly decreased p53 acetylation, thereby inhibiting mitochondrial apoptosis $[16,17]$. Recently, resveratrol has been reported to enhance cell survival and inhibit apoptosis by inhibiting p53 acetylation via the SIRT1 signaling pathway [13].

3,4,5-Trihydroxycinnamic acid (THC), a hydroxycinnamic acid derivative, was originally identified in Rooibos tea [18] and has been reported to exhibit a wide range of pharmacological properties, including anti-inflammatory and neuroprotective properties [19-21]. Caffeic acid phenethyl ester, a hydroxycinnamic ester derivative, has been demonstrated to suppress cytokine-induced NF- $\kappa B$ signaling in macrophage cells [22] and plays important roles in the regulation of the peripheral immune response through the activation of the nuclear factor erythroid 2-related factor/heme oxygenase-1 (Nrf2/HO-1) signaling cascade [23]. We observed that THC significantly in-

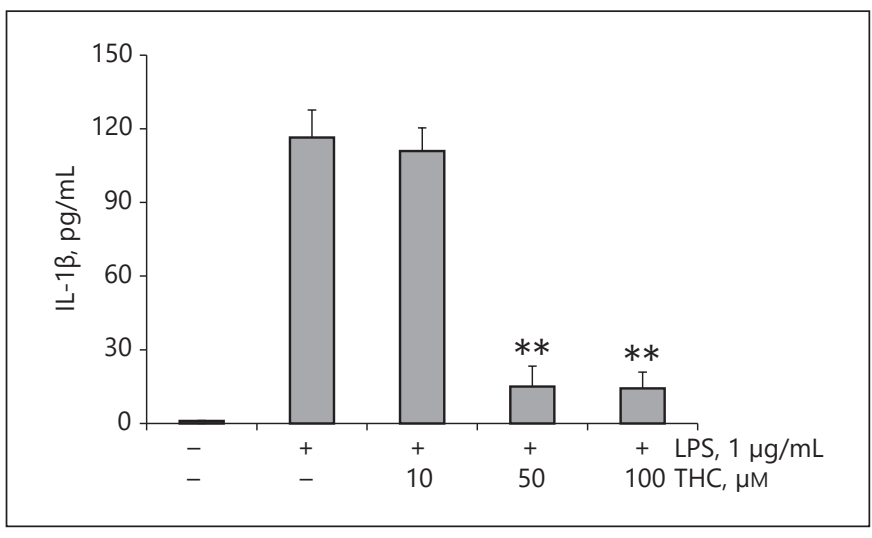

Fig. 1. THC inhibits LPS-induced IL- $1 \beta$ production. HUVECs were treated with $1 \mu \mathrm{g} / \mathrm{mL}$ LPS in the absence or presence of THC $(10,50$, and $100 \mu \mathrm{M})$. IL-1 $\beta$ levels in culture supernatants were measured by ELISA. THC significantly suppressed the LPS-induced production of IL- $1 \beta$ in a concentration-dependent manner. The values are expressed as the mean \pm SD of 3 independent experiments. ${ }^{* *} p<0.01$ versus LPS alone. THC, 3,4,5-trihydroxycinnamic acid; HUVECs, human umbilical vein endothelial cells; IL, interleukin; SD, standard deviation.

hibited LPS-induced upregulation of pro-inflammatory mediators through the inhibition of NF- $\kappa B$ activation in microglial cells [24]. Recently, we also identified that THC inhibited LPS-induced inflammatory responses through the expression of HO-1 via $\mathrm{Nrf} 2$ activation in macrophage cells and improved the survival of mice in an LPS-induced endotoxemia model $[25,26]$. However, the effect of THC on vascular endothelial cells and the underlying mechanism have not been clearly examined. Therefore, the goal of the present study was to examine the anti-inflammatory properties of THC on vascular endothelial cells and its underlying mechanism in human umbilical vein endothelial cells (HUVECs) in order to provide an important therapeutic agent that could attenuate pathologically activated endothelial cells in various inflammatory conditions, including sepsis.

\section{Materials and Methods}

\section{Reagents and Cell Culture}

Bacterial LPS and Escherichia coli serotype 055:B5 were obtained from Sigma-Aldrich (St. Louis, MO, USA). THC was obtained from AApin Chemicals Ltd. (Abingdon, UK). THC, solubilized in dimethyl sulfoxide (DMSO), was added to the culture media at desired concentrations. HUVECs were obtained from American Type Culture Collection (ATCC; CRL-1730) and grown on 2\% gelatin-coated plates in M199 (Hyclone Laboratories) supplemented with $20 \%$ fetal bovine serum, $3 \mathrm{ng} / \mathrm{mL}$ basic fibroblast 


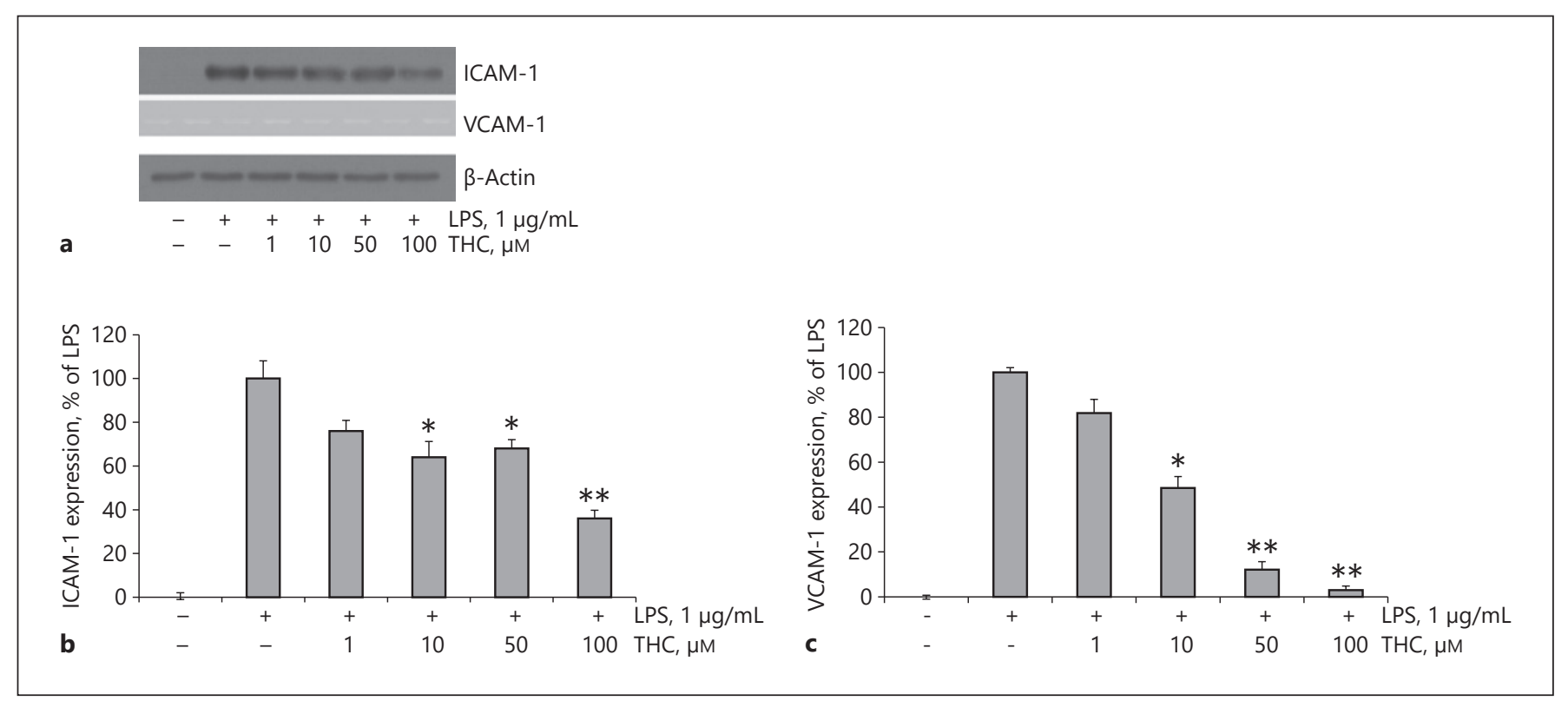

Fig. 2. THC inhibits LPS-induced ICAM-1 and VCAM-1 expression. HUVECs were pretreated with THC $(10,50$, and $100 \mu \mathrm{M})$ for $24 \mathrm{~h}$ and then stimulated with $1 \mu \mathrm{g} / \mathrm{mL}$ LPS for $24 \mathrm{~h}$. The expression of ICAM-1 and VCAM-1 was measured by Western blotting. THC significantly suppressed the LPS-induced upregulation of ICAM-1 and VCAM-1 in a concentration-dependent manner. The data presented are the mean \pm SD of 3 independent experi-

growth factor (bFGF), $100 \mathrm{U} / \mathrm{mL}$ penicillin-streptomycin (Gibco $\mathrm{BRL}$ ), and $5 \mathrm{U} / \mathrm{mL}$ heparin (culture medium) in a humidified $5 \%$ $\mathrm{CO}_{2}$ incubator. For experiments, cells between passages 2 and 6 were cultured on gelatin-coated 6 -well plates and incubated for $6 \mathrm{~h}$ in M199 supplemented with 1\% fetal bovine serum and $100 \mathrm{U} /$ $\mathrm{mL}$ penicillin-streptomycin (starvation medium). Cells were incubated with the indicated concentrations of THC before treatment with LPS $(1 \mu \mathrm{g} / \mathrm{mL})$.

Cytokine Assays

HUVECs were pretreated with THC $(1-100 \mu \mathrm{M})$ for $24 \mathrm{~h}$ and then challenged with or without LPS $(1 \mu \mathrm{g} / \mathrm{mL})$ for $24 \mathrm{~h}$. Interleukin (IL)- $1 \beta$ released into the culture media of HUVECs was measured using ELISA kits (R\&D system, Minneapolis, MN, USA) according to the manufacturer's instructions.

\section{Western Blot Analysis}

HUVECs were incubated with THC for $3 \mathrm{~h}$ before LPS challenge. HUVECs were washed with ice-cold PBS and lysed in PROPREP lysis buffer (iNtRON Biotechnology, Seongnam, Korea). Equal amounts of protein lysates were separated on a 10\% SDSpolyacrylamide gel. Proteins were then transferred to Hybond PVDF membranes (Amersham Biosciences, Piscataway, NJ, USA) and blocked in 5\% skim milk in TBST for $1 \mathrm{~h}$ at room temperature. Specific antibodies against intercellular adhesion molecule-1 (ICAM-1), vascular cell adhesion molecule-1 (VCAM-1), SIRT1 (1:1,000; Santa Cruz Biotechnology Inc.), p65, p-p65, IкB, p-IкB, ments. ${ }^{*} p<0.05,{ }^{* *} p<0.01$ versus LPS alone. a Representative immunoblots of ICAM-1 and VCAM-1. b, c Quantitative analyses of immunoblots of ICAM-1 and VCAM-1, respectively. THC, 3,4,5-trihydroxycinnamic acid; ICAM-1, intercellular adhesion molecule-1; VCAM-1, vascular cell adhesion molecule-1; SD, standard deviation.

p53, acetyl-p53, FOXO3a, acetyl-FOXO3a, PGC1a, acetyl-PGC1a (1:1,000; Cell Signaling Technology), and $\beta$-actin (1:2,500; Sigma) were diluted in 5\% TBST-milk. After thorough washing with TBST, appropriate HRP-conjugated secondary antibodies were added. The blots were then developed and detected using an enhanced chemiluminescence agent (Amersham Biosciences).

\section{Statistical Analysis}

All experimental values shown in the figures are denoted as the mean \pm SD, which were obtained from at least 3 independent experiments. Statistical differences were analyzed by one-way ANOVA with Tukey's post hoc test using SPSS software 12K (SPSS, Chicago, IL, USA). Data with values of $p<0.05$ were considered statistically significant. Single $(*)$ and double $\left({ }^{* *}\right)$ marks represent statistical significance at $p<0.05$ and $p<0.01$, respectively.

\section{Results}

\section{THC Inhibits IL-1 $\beta$ Secretion in LPS-Challenged HUVECS}

Previous studies have shown that inflammatory cytokines such as IL-1 $\beta$ play a key role in the progression of inflammation $[27,28]$. Here, we examined the effects of THC on the extracellular release of IL-1 $\beta$ in LPS-chal- 


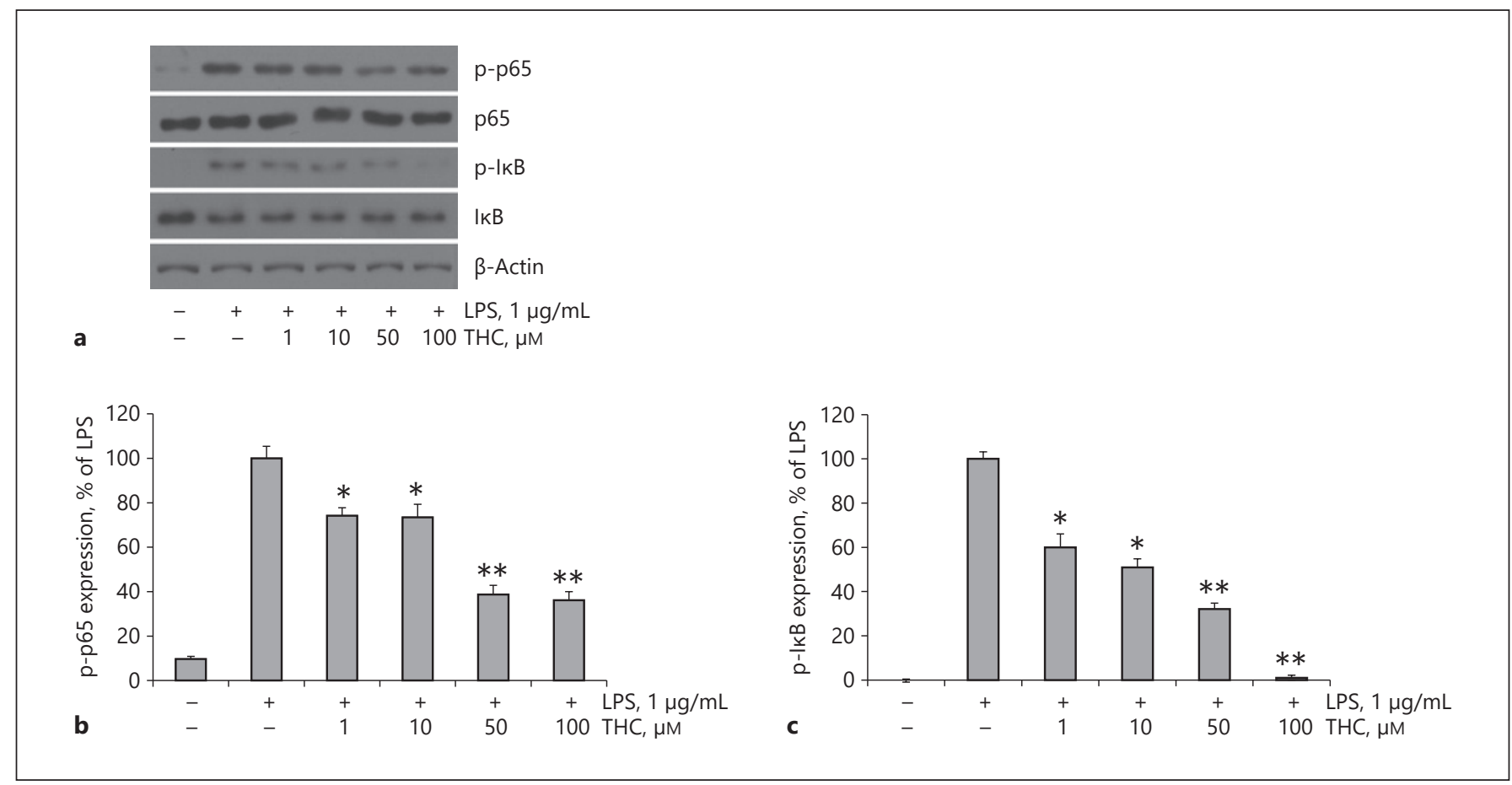

Fig. 3. THC inhibits LPS-induced NF- $\kappa B$ activation and I $\kappa \mathrm{B}$ degradation. HUVECs were pretreated with $\operatorname{THC}(1,10,50$, and 100 $\mu \mathrm{M})$ for $3 \mathrm{~h}$ and then stimulated with $1 \mu \mathrm{g} / \mathrm{mL}$ LPS for $24 \mathrm{~h}$. THC significantly suppressed the LPS-induced phosphorylation of p65 and $\mathrm{I} \kappa \mathrm{B}$ in a concentration-dependent manner. The data presented are the mean \pm SD of 3 independent experiments. ${ }^{*} p<0.05$,

lenged HUVECs. Cells were incubated with THC for 24 $\mathrm{h}$ prior to LPS treatment $(1 \mu \mathrm{g} / \mathrm{mL})$. LPS increased the production of IL- $1 \beta$ in HUVECs. THC significantly suppressed the extracellular release of IL- $1 \beta$ in LPS-stimulated HUVECs at concentrations of $50 \mu \mathrm{M}$ and above, which was almost 6-fold lower than that of the control (Fig. 1). No noticeable cytotoxicity was observed with THC in the concentration ranges used in the present study (data not shown).

THC Inhibits LPS-Induced Expression of ICAM-1 and VCAM-1

It has been widely reported that upregulation of VCAMs plays a pivotal role in the aggravation of inflammatory conditions, including sepsis [7]. In the present study, LPS treatment significantly increased the expression of the ICAM- 1 and VCAM- 1 adhesion proteins in HUVECs. THC treatment resulted in significantly suppressed expression of ICAM-1 and VCAM-1 from $10 \mu \mathrm{M}$ in a concentration-dependent manner in
${ }^{* *} p<0.01$ versus LPS alone. a Representative immunoblots of phosphorylation levels of p65 and IкB. b, c Quantitative analyses of immunoblots of p-p65 and p-IкB, respectively. $\beta$-Actin was used as a control. THC, 3,4,5-trihydroxycinnamic acid; NF- $\mathrm{kB}$, nuclear factor- $\kappa B$; I $\mathrm{KB}$, inhibitor of kappa B; HUVECs, human umbilical vein endothelial cells.

LPS-challenged HUVECs (Fig. 2), suggesting that THC might attenuate endothelial dysfunction in inflammatory conditions.

\section{THC Inhibits Phosphorylation of $p 65$ and}

Degradation of $I \kappa B$

In the present study, the effect of THC on LPS-induced NF- $\kappa B$ activation was examined in HUVECs as NF- $\kappa B$ is a key transcription factor of pro-inflammatory genes and I $\kappa \mathrm{B}$ inhibits nuclear translocation of the phosphorylated p65 subunit of NF- $\mathrm{BB}$ [28]. LPS challenge showed significantly increased phosphorylation of the p65 subunit of NF- $\kappa B$. THC treatment significantly attenuated LPSinduced phosphorylation of the p65 subunit in a concentration-dependent manner (Fig. 3). In addition, THC significantly suppressed LPS-induced degradation of I $\mathrm{B}$ in a concentration-dependent manner (Fig. 3) with significant inhibition that started at $1 \mu \mathrm{M}$. Thus, THC inhibited LPS-induced NF- $\kappa B$ activation by suppression of $\mathrm{p} 65$ phosphorylation and I $\kappa \mathrm{B}$ degradation. 


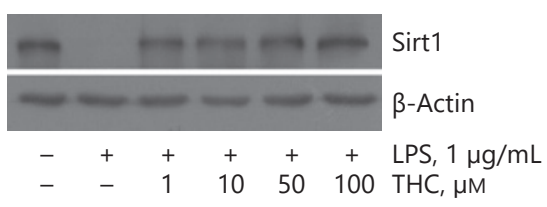

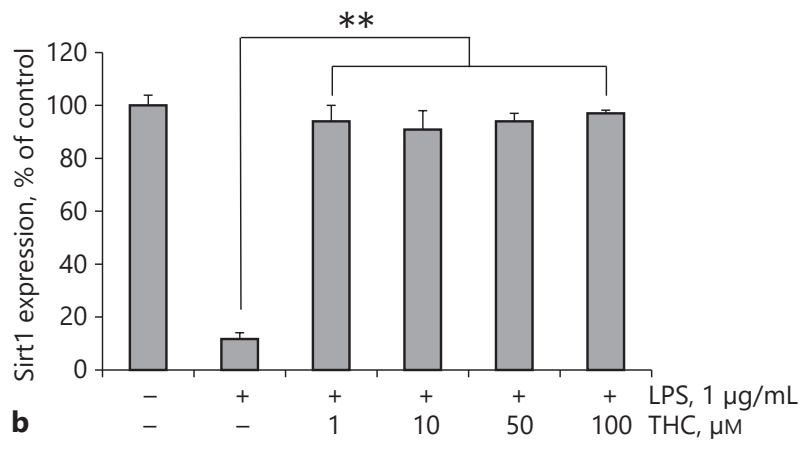

ments. ${ }^{*} p<0.05,{ }^{* *} p<0.01$ versus LPS alone. Representative image of SIRT1 expression (a) and quantitative analysis of immunoblots (b). $\beta$-Actin was used as a control. THC, 3,4,5-trihydroxycinnamic acid; HUVECs, human umbilical vein endothelial cells; SIRT1, sirtuin 1 .

\section{sirtuin 1.}

Fig. 4. THC inhibits LPS-induced suppression of SIRT1 expression. HUVECs were pretreated with THC $(1,10,50$, and $100 \mu \mathrm{M})$ for $3 \mathrm{~h}$ and then stimulated with $1 \mu \mathrm{g} / \mathrm{mL}$ LPS for $24 \mathrm{~h}$. THC significantly restored LPS-induced suppression of SIRT1 expression. The data presented are the mean \pm SD of 3 independent experi-

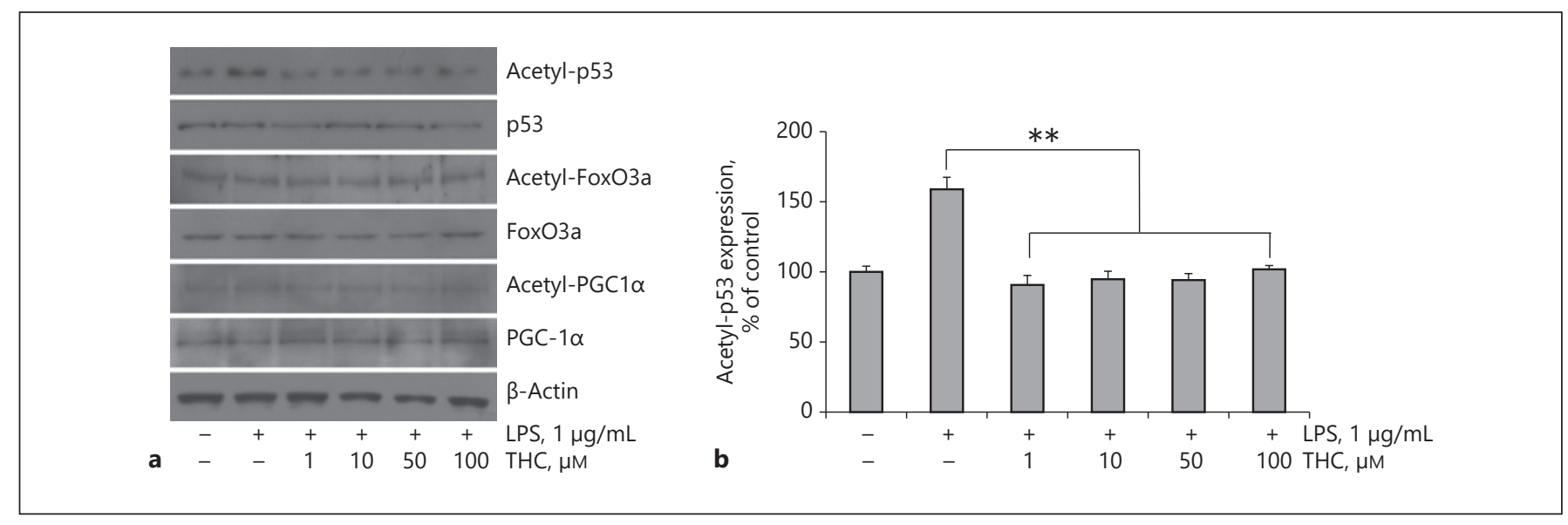

Fig. 5. THC inhibits LPS-induced acetylation of p53. HUVECs were pretreated with $\operatorname{THC}(1,10,50$, and $100 \mu \mathrm{M})$ for $3 \mathrm{~h}$ and then stimulated with $1 \mu \mathrm{g} / \mathrm{mL}$ LPS for $24 \mathrm{~h}$. THC significantly suppressed LPS-induced increase in acetylated p53. However, noticeable changes in the acetylation of other SIRT1 downstream molecular targets, such as FOXO3a and PGC1a, were not observed. a Representative immunoblots of acetylation levels of SIRT1

\section{THC Induces SIRT1 Expression in LPS-Challenged HUVECS}

SIRT1 has been reported to be involved in the regulatory control of a wide range of cellular processes, such as cell survival, DNA repair, and cell migration [13]. The effect of THC on the expression of SIRT1 was examined in the present study. LPS challenge resulted in decreased expression of SIRT1 in HUVECs, and THC treatment sig- downstream targets. b Quantitative analyses of immunoblots of acetylated p53. The data presented are the mean \pm SD of 3 independent experiments. ${ }^{*} p<0.05,{ }^{*} p<0.01$ versus LPS alone. $\beta$-Actin was used as a control. THC, 3,4,5-trihydroxycinnamic acid; HUVECs, human umbilical vein endothelial cells; SIRT1, sirtuin 1; FOXO3a, forkhead box O3; PGC1 $\alpha$, peroxisome proliferator activated receptor gamma coactivator 1 alpha.

nificantly abolished the LPS-induced suppression of SIRT1 (Fig. 4a) at concentrations of $1 \mu \mathrm{M}$ and above. However, the recovery by THC was not concentration dependent (Fig. 4b). In addition, THC treatment in the absence of LPS challenge did not result in increased expression of SIRT1 (Fig. 6a). Quantitative analysis showed no significant changes in SIRT1 level in the absence of LPS challenge (data not shown), suggesting that THC- 


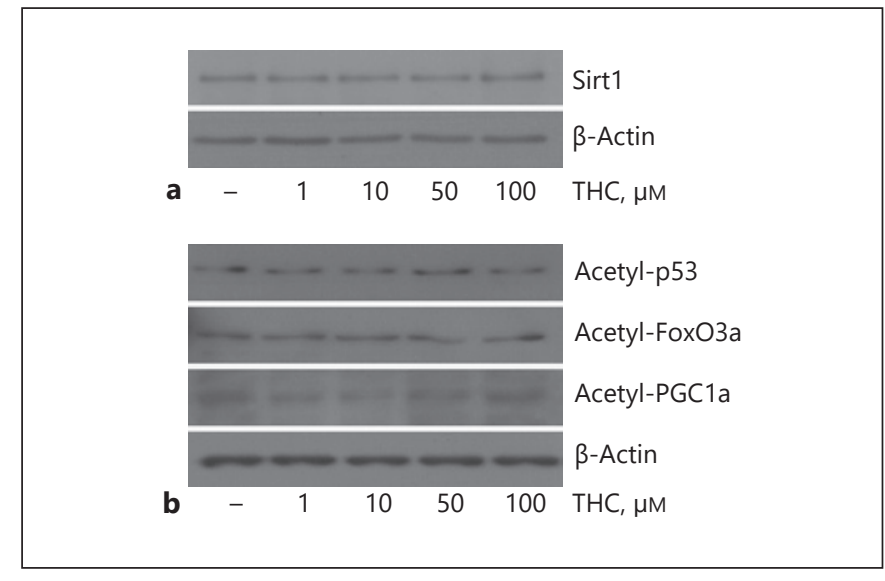

Fig. 6. THC does not induce the expression of SIRT1 and subsequent inhibition of acetylation of downstream targets in the absence of LPS challenge. HUVECs were pretreated with THC $(1,10$, 50 , and $100 \mu \mathrm{M}$ ) for $3 \mathrm{~h}$ and then stimulated with $1 \mu \mathrm{g} / \mathrm{mL}$ LPS for $24 \mathrm{~h}$. THC did not exhibit the upregulation of SIRT1 and subsequent suppression of downstream targets in the absence of LPS challenge. Representative images of SIRT1 expression (a) and levels of acetylated p53, FOXO3a, and PGC1a (b). Images are representative of 3 independent experiments that show reproducible results. $\beta$-Actin was used as a control. THC, 3,4,5-trihydroxycinnamic acid; HUVECs, human umbilical vein endothelial cells; SIRT1, sirtuin 1; FOXO3a, forkhead box O3; PGC1a, peroxisome proliferator activated receptor gamma coactivator 1 alpha.

mediated expression of SIRT1 might be LPS challenge dependent.

\section{THC Suppresses Acetylated p53 in LPS-Challenged HUVECS}

Given the previous reports that SIRT1 exerts its diverse cellular functions through downstream molecular targets such as p53, FOXO3a, and PGC1a [13], the expression of these proteins was examined to determine the effect of THC on these downstream targets in the present study. LPS challenge showed an increased level of acetylated p53, and THC treatment significantly suppressed the LPS-induced acetylation of p53 (Fig. 5). However, in accordance with the result that a negligible effect of THC was observed on SIRT1 levels in the absence of LPS challenge (Fig. 6a), THC did not exhibit significant attenuation of p53 acetylation in the absence of LPS challenge (Fig. 6b), suggesting that THC-mediated suppression of p53 acetylation might be LPS challenge dependent. In addition, noticeable changes were not observed with the levels of FOXO3a and PGCla in the present study (Fig. 5).

THC-Induced SIRT1 Expression in HUVECs

\section{Discussion}

Our results demonstrate that THC significantly attenuates LPS-induced inflammatory responses in vascular endothelial cells. THC significantly attenuated LPS-induced secretion of IL- $1 \beta$ and expression of ICAM- 1 and VCAM-1 in LPS-challenged HUVECs. THC significantly attenuated LPS-induced NF- $\kappa$ B activation and resulted in the activation of SIRT1 signaling with subsequent inactivation of $\mathrm{p} 53$.

Aberrant endothelial activation has been reported in sepsis and in many cardiovascular disorders, such as hypertension, atherosclerosis, and hemolysis [29, 30]. Vascular endothelial cells overproduce various cytokines and mediators during the inflammatory response, which aggravates the inflammatory damage [31]. In our study, LPS-induced overproduction of IL- $1 \beta$ was significantly inhibited by THC treatment (Fig. 1). The vascular endothelium provides a barrier that selectively restricts the passage of plasma and cells from the blood into the adjacent tissue [32]. Previously, we observed that LPS induced increased vascular leakage in multiple organs, including the kidney, liver, and spleen, and that THC significantly suppressed this leakage in an LPS-induced septic animal model [9]. In addition, THC significantly attenuated LPS-induced macrophage infiltration into the kidney in an LPS-induced septic mouse model [33].

A characteristic feature of vascular endothelial dysfunction is the adhesion of immune cells to the endothelium, which is controlled by the expression of cell adhesion molecules such as ICAM-1 and VCAM-1 $[34,35]$. It has been reported that LPS induces the adhesion of neutrophils to endothelial cells $[36,37]$. The present study demonstrates that THC significantly suppressed LPS-induced expression of ICAM-1 and VCAM-1 in a concentration-dependent manner in HUVECs (Fig. 2). This implies that THC might attenuate the tissue infiltration of immune cells by suppressing their adhesion to endothelial cells.

$\mathrm{NF}-\mathrm{\kappa B}$ is the primary transcription factor involved in inflammatory responses [28]. It is well known that NF- $\kappa B$ is involved in the production of pro-inflammatory mediators, such as inducible nitric oxide synthase, IL-1 $\beta$, and tumor necrosis factor, and the expression of cell adhesion molecules, such as ICAM- 1 and VCAM-1 $[38,39]$. LPS causes the phosphorylation of the 665 subunit of NF$\kappa \mathrm{B}$ and subsequent nuclear translocation of the phosphorylated NF- $\kappa B$ through I $\kappa B$ degradation $[25,26]$. Our results show that LPS-induced phosphorylation of p65 is significantly attenuated by THC treatment in a concen- 
tration-dependent manner in endothelial cells (Fig. 3). In addition, THC significantly inhibited LPS-mediated IкB phosphorylation in a dose-dependent manner (Fig. 3), resulting in the significant inhibition of LPS-induced nuclear translocation of NF- $\kappa \mathrm{B}$.

SIRT1 is an NAD-dependent class III histone deacetylase that plays key physiological roles in enhancing cell survival and suppressing apoptosis [40]. SIRT1 has also been demonstrated to control inflammatory mediator production and activation via the inhibition of NF- $\kappa B$ $[41,42]$. The present study demonstrated that THC significantly attenuated LPS-induced NF- $\kappa \mathrm{B}$ activation in HUVECs (Fig. 3) and that THC abolished the LPS-induced downregulation of SIRT1 expression (Fig. 4). It has been previously reported that activated NF- $\kappa \mathrm{B}$ down-regulates SIRT1 signaling through the expression of a variety of mediators, such as miR-34a, interferon gamma, and reactive oxygen species, by deacetylating the $\mathrm{p} 65$ subunit of NF- $\kappa B$ [43]. It has been reported that THC attenuates phosphorylation of NF- $\mathrm{KB}$ in microglial cells [28] and septic kidney cells [33]. Therefore, SIRT1 activity in the present study might be indirectly due to THC-mediated suppression of NF- $\mathrm{KB}$. Although SIRT1 suppression of NF- $\kappa B$ signaling through the deacetylation of the p65 subunit of NF- $\kappa B$ has been shown [43], the present study lacks evidence of SIRT1-mediated deacetylation of NF$\kappa B$. In addition, SIRT1 inhibitor or knockout/knockdown studies should be applied to clearly demonstrate the role of SIRT1 in THC-mediated anti-inflammatory action. Further studies are necessary to clearly elucidate the role of THC in the antagonistic crosstalk between NF- $\kappa \mathrm{B}$ and SIRT1 and the role of SIRT1 in THC-mediated anti-inflammatory action. Upregulation of SIRT1 by THC was not observed in the absence of LPS challenge (Fig. 6a), suggesting that THC-mediated upregulation of SIRT1 expression might be a stress-dependent phenomenon. SIRT1 regulates the functions of various downstream factors by directly deacetylating targets, including p53, FOXO3a, PGC1 $\alpha$, and NF- $\mathrm{BB}$ [44]. In this study, THC significantly decreases the LPS-induced increase in the level of acetylated p53 in HUVECs (Fig. 5). However, the attenuation of acetylation of p53 was not concentration dependent, which is in accordance with the level of SIRT1 in LPS-challenged HUVECs. In addition, the change in the acetylation levels of FOXO3a and PGC1 $\alpha$ was not noticeable with LPS challenge in the present study, and THC-mediated changes in the acetylation levels of these 2 proteins were not observed (Fig. 5). It has been widely reported that activation of p53 through acetylation is involved in apoptosis and cell death $[14,15]$. Enhanced ex- pression of SIRT1 has been reported to decrease p53 acetylation and inhibition of apoptosis $[16,17]$. This study shows that THC restores LPS-induced downregulation of SIRT1 and suppresses the LPS-induced increase in acetylated p53 in HUVECs (Fig. 5). This is in accordance with previous reports that resveratrol enhanced cell survival and inhibited apoptosis through SIRT1-mediated inhibition of p53 acetylation [13].

Hydroxycinnamic acids and their ester derivatives have been demonstrated to exhibit a wide range of biological properties, such as anticancer, anti-inflammatory, immunosuppressive, antimicrobial, and neuroprotective properties [19-21]. THC exhibited anti-inflammatory properties through the suppression of NF- $\kappa \mathrm{B}$ activation in LPS-challenged BV2 microglial cells [28]. We reported that THC resulted in the suppression of LPS-induced inflammatory response through the activation of the Nrf2/ HO-1 signaling pathway in RAW264.7 macrophage cells [9]. THC suppressed LPS-induced macrophage infiltration into the kidney and exhibited improved survival in mice in the LPS-induced endotoxemia model $[9,33]$. The present data demonstrate that THC significantly suppresses LPS-induced expression of the adhesion proteins ICAM-1 and VCAM-1 (Fig. 2). This suggests that THCmediated inhibition of endothelial cell adhesion molecules might contribute to the improved survival of septic mice through the suppression of macrophage infiltration to various organs.

In conclusion, in addition to our previous reports that THC exerts anti-inflammatory responses in BV2 microglial cells $[9,33]$ and endotoxemia animal models $[9,33]$, the present study clearly shows that THC inhibits LPSinduced inflammatory responses through the inactivation of the NF- $\kappa \mathrm{B}$ signaling pathway, the upregulation of SIRT1 expression, and the subsequent inhibition of p53 signaling in HUVECs. Therefore, THC may provide an important therapeutic option in the treatment of inflammation-related endothelial dysfunction in various vascular disorders.

\section{Acknowledgement}

This study was supported by a 2017 Research Grant from Kangwon National University (No. 520170442).

\section{Statement of Ethics}

The authors complied with internationally accepted standards for research practice and reporting. 


\section{Disclosure Statement}

The authors have no conflicts of interest.

\section{Author Contributions}

J.P. performed experiments, and analyzed and interpreted data under the guidance of W.C., H.L., and Y.K., who provided suggestions for interpreting the results of the study. All authors have contributed to, seen, read, and approved the manuscript.

\section{References}

1 Nomura J, Busso N, Ives A, Matsui C, Tsujimoto $\mathrm{S}$, Shirakura T, et al. Xanthine oxidase inhibition by febuxostat attenuates experimental atherosclerosis in mice. Sci Rep. 2014 Apr 1;4:4554.

2 Gray SP, Jandeleit-Dahm KA. The role of $\mathrm{NADPH}$ oxidase in vascular disease: hypertension, atherosclerosis \& stroke. Curr Pharm Des. 2015;21(41):5933-44.

3 Han JM, Li H, Cho MH, Baek SH, Lee CH, Park HY, et al. Soy-leaf extract exerts atheroprotective effects via modulation of Krüppellike factor 2 and adhesion molecules. Int J Mol Sci. 2017 Feb 10;18(2):373.

4 Kang JS, Jeon YJ, Park SK, Yang KH, Kim HM. Protection against lipopolysaccharideinduced sepsis and inhibition of interleukin1beta and prostaglandin E2 synthesis by silymarin. Biochem Pharmacol. 2004 Jan 1; 67(1):175-81.

5 Reinhart K, Bayer O, Brunkhorst F, Meisner M. Markers of endothelial damage in organ dysfunction and sepsis. Crit Care Med. 2002 May;30(5 Suppl):S302-12.

6 Yano K, Liaw PC, Mullington JM, Shih SC, Okada H, Bodyak N, et al. Vascular endothelial growth factor is an important determinant of sepsis morbidity and mortality. J Exp Med. 2006 Jun 12;203(6):1447-58.

7 Shapiro NI, Khankin EV, Van Meurs M, Shih SC, Lu S, Yano M, et al. Leptin exacerbates sepsis-mediated morbidity and mortality. J Immunol. 2010 Jul 1;185(1):517-24.

8 Lee HJ, Lim HJ, Lee DY, Jung H, Kim MR, Moon DC, et al. Carabrol suppresses LPS-induced nitric oxide synthase expression by inactivation of $\mathrm{p} 38$ and JNK via inhibition of I- $\mathrm{kBa}$ degradation in RAW 264.7 cells. Biochem Biophys Res Commun. 2009 Jan 15;391(3):1400-4.

9 Lee JW, Bae CJ, Choi YJ, Kim SI, Kwon YS, Lee HJ, et al. 3,4,5-Trihydroxycinnamic acid inhibits lipopolysaccharide (LPS)-induced inflammation by Nrf2 activation in vitro and improves survival of mice in LPS-induced endotoxemia model in vivo. Mol Cell Biochem. 2014 May;390(1-2):143-53.

10 Yi J, Luo J. SIRT1 and p53, effect on cancer, senescence and beyond. Biochim Biophys Acta. 2010 Aug;1804(8):1684-9.

11 Ben Salem I, Boussabbeh M, Pires Da Silva J, Guilbert A, Bacha H, Abid-Essefi S, et al. SIRT1 protects cardiac cells against apoptosis induced by zearalenone or its metabolites $\alpha$ and $\beta$-zearalenol through an autophagy-dependent pathway. Toxicol Appl Pharmacol. 2016 Jan 1;314:82-90.
12 Wang XL, Wu LY, Zhao L, Sun LN, Liu HY, Liu G, et al. SIRT1 activator ameliorates the renal tubular injury induced by hyperglycemia in vivo and in vitro via inhibiting apoptosis. Biomed Pharmacother. 2016 Oct;83:4150.

13 Liu H, Zhang S, Liu C, Wu J, Wang Y, Yuan $\mathrm{L}$, et al. Resveratrol ameliorates microcystinLR-induced testis germ cell apoptosis in rats via SIRT1 signaling pathway activation. Toxins. 2018 Jun 9;10(6):235.

14 Miyashita T, Krajewski S, Krajewska M, Wang HG, Lin HK, Liebermann DA, et al. Tumor suppressor $\mathrm{p} 53$ is a regulator of bcl-2 and bax gene expression in vitro and in vivo. Oncogene. 1994 Jun;9(6):1799-805.

15 Kannan K, Amariglio N, Rechavi G, JakobHirsch J, Kela I, Kaminski N, et al. DNA microarrays identification of primary and secondary target genes regulated by $\mathrm{p} 53$. Oncogene. 2001 Apr 26;20(18):2225-34.

16 Yan S, Wang M, Zhao J, Zhang H, Zhou C, Jin L, et al. MicroRNA-34a affects chondrocyte apoptosis and proliferation by targeting the SIRT1/p53 signaling pathway during the pathogenesis of osteoarthritis. Int J Mol Med. 2016 Jul;38(1):201-9.

17 Zhang F, Zhang M, Wang A, Xu M, Wang C, $\mathrm{Xu} \mathrm{G}$, et al. TWEAK increases SIRT1 expression and promotes p53 deacetylation affecting human hepatic stellate cell senescence. Cell Biol Int. 2016 Feb;41(2):147-54.

18 Rabe C, Steenkamp JA, Joubert E, Burger JFW, Ferreira D. Phenolic metabolites from rooibos tea (Aspalathus linearis). Phytochemistry. 1994;35(6):1559-65.

19 Nagasaka R, Chotimarkorn C, Shafiqul IM, Hori M, Ozaki H, Ushio H. Anti-inflammatory effects of hydroxycinnamic acid derivatives. Biochem Biophys Res Commun. 2007 Jun 29;358(2):615-9.

20 Kim YC. Neuroprotective phenolics in medicinal plants. Arch Pharm Res. 2010 Oct; 33(10):1611-32.

21 Lee JW, Cheong IY, Kim HS, Lee JJ, Lee YS, Kwon YS, et al. Anti-inflammatory activity of 1-docosanoyl Cafferate Isolated from Rhus verniciflua in LPS-stimulated BV2 Microglial Cells. Korean J Physiol Pharmacol. 2011 Feb; 15(1):9-15.

22 Lee Y, Shin DH, Kim JH, Hong S, Choi D, Kim YJ, et al. Caffeic acid phenethyl ester-mediated Nrf2 activation and I $\mathrm{KB}$ kinase inhibition are involved in NFKB inhibitory effect: structural analysis for NFkB inhibition. Eur J Pharmacol. 2010 Sep 15;643(1):21-8.
23 Fidan H, Sahin O, Yavuz Y, Kilbas A, Cetinkaya Z, Ela Y, et al. Caffeic acid phenethyl ester reduces mortality and sepsis-induced lung injury in rats. Crit Care Med. 2007 Dec; 35(12):2822-9.

24 Itharat A, Hiransai P. Dioscoreanone suppresses LPS-induced nitric oxide production and inflammatory cytokine expression in RAW 264.7 macrophages by NF- $\mathrm{kB}$ and ERK1/2 signaling transduction. J Cell Biochem. 2012 Nov;113(11):3427-35.

25 Kim YJ, Shin Y, Lee KH, Kim TJ. Anethum graveloens flower extracts inhibited a lipopolysaccharide-induced inflammatory response by blocking iNOS expression and NF$\kappa \mathrm{B}$ activity in macrophages. Biosci Biotechnol Biochem. 2012;76(6):1122-7.

26 Rehman MU, Yoshihisa Y, Miyamoto Y, Shimizu T. The anti-inflammatory effects of platinum nanoparticles on the lipopolysaccharide-induced inflammatory response in RAW 264.7 macrophages. Inflamm Res. 2012 Nov; 61(11):1177-85.

27 Ock J, Kim S, Suk K. Anti-inflammatory effects of a fluorovinyloxyacetamide compound KT-15087 in microglia cells. Pharmacol Res. 2009 Jun;59(6):414-22.

28 Lee JW, Bae CJ, Choi YJ, Kim SI, Kim NH, Lee HJ, et al. 3,4,5-Trihydroxycinnamic acid inhibits LPS-induced iNOS expression by suppressing NF- $\kappa B$ activation in BV2 microglial cells. Korean J Physiol Pharmacol. 2012 Apr; 16(2):107-12.

29 Ross R. Atherosclerosis: an inflammatory disease. N Engl J Med. 1999 Jan 14;340(2):115-26.

30 Bains SK, Foresti R, Howard J, Atwal S, Green CJ, Motterlini R. Human sickle cell blood modulates endothelial heme oxygenase activity: effects on vascular adhesion and reactivity. Arterioscler Thromb Vasc Biol. 2009 Feb; 30(2):305-12.

31 Liu XH, Pan LL, Yang HB, Gong QH, Zhu YZ. Leonurine attenuates lipopolysaccharide-induced inflammatory responses in human endothelial cells: involvement of reactive oxygen species and NF- $\kappa B$ pathways. Eur J Pharmacol. 2012 Apr 5;680(1-3):108-14.

32 Fisher M. Injuries to the vascular endothelium: vascular wall and endothelial dysfunction. Rev Neurol Dis. 2008;5(Suppl 1):S4-11.

33 Lee JW, Kwon JH, Lim MS, Lee HJ, Kim SS, Lim SY, et al. 3,4,5-Trihydroxycinnamic acid increases heme-oxygenase-1 (HO-1) and decreases macrophage infiltration in LPS-induced septic kidney. Mol Cell Biochem. 2014 Dec;397(1-2):109-16. 
34 Su Y, Lei X, Wu L, Liu L. The role of endothelial cell adhesion molecules P-selectin, E-selectin and intercellular adhesion molecule-1 in leucocyte recruitment induced by exogenous methylglyoxal. Immunology. 2012 Sep; 137(1):65-79.

35 Kim JH, Yoon YJ, Lee J, Choi EJ, Yi N, Park KS, et al. Outer membrane vesicles derived from Escherichia coli up-regulate expression of endothelial cell adhesion molecules in vitro and in vivo. PLoS One. 2013;8(3):e59276.

$36 \mathrm{Kim} \mathrm{TH,} \mathrm{Ku} \mathrm{SK,} \mathrm{Lee} \mathrm{IC,} \mathrm{Bae} \mathrm{JS.} \mathrm{Anti-inflam-}$ matory effects of kaempferol-3-O-sophoroside in human endothelial cells. Inflamm Res. 2011 Mar;61(3):217-24.

37 Li Y, Zhang XS, Yu JL. Acanthoic acid inhibits LPS-induced inflammatory response by activating LXR $\alpha$ in human umbilical vein endothelial cells. Int Immunopharmacol. 2016 Mar;32:111-5.
38 Wan M, Liu J, Ouyang X. Nucleotide-binding oligomerization domain 1 regulates porphyromonas gingivalis-induced vascular cell adhesion molecule 1 and intercellular adhesion molecule 1 expression in endothelial cells through NF- $\kappa \mathrm{B}$ pathway. J Periodont Res. 2014 Apr;50(2):189-96.

39 Wang L, Xu Y, Yu Q, Sun Q, Xu Y, Gu Q, et al. H-RN, a novel antiangiogenic peptide derived from hepatocyte growth factor inhibits inflammation in vitro and in vivo through $\mathrm{PI} 3 \mathrm{~K} / \mathrm{AKT} / \mathrm{IKK} / \mathrm{NF}-\kappa \mathrm{B}$ signal pathway. Biochem Pharmacol. 2014 May 15;89(2):255-65.

40 Jang J, Huh YJ, Cho HJ, Lee B, Park J, Hwang DY, et al. SIRT1 enhances the survival of human embryonic stem cells by promoting DNA repair. Stem Cell Reports. 2017 Aug 8; 9(2):629-41.
41 Zhu X, Liu Q, Wang M, Liang M, Yang X, Xu $X$, et al. Activation of Sirtl by resveratrol inhibits TNF- $\alpha$ induced inflammation in fibroblasts. PLoS One. 2011;6(11):e27081.

42 Moon MH, Jeong JK, Lee YJ, Seol JW, Jackson CJ, Park SY. SIRT1, a class III histone deacetylase, regulates TNF- $\alpha$-induced inflammation in human chondrocytes. Osteoarthr Cartil. 2012 Mar;21(3):470-80.

43 Kauppinen A, Suuronen T, Ojala J, Kaarniranta K, Salminen A. Antagonistic crosstalk between NF- $\kappa B$ and SIRT1 in the regulation of inflammation and metabolic disorders. Cell Signal. 2013;25(10):1939-48.

44 Chen Z, Shentu TP, Wen L, Johnson DA, Shyy JY. Regulation of SIRT1 by oxidative stress-responsive miRNAs and a systematic approach to identify its role in the endothelium. Antioxid Redox Signal. 2013 Nov 1; 19(13):1522-38. 